\title{
Standardization of Cytokinins (BAP and Kinetin) Concentrations and their Combination with NAA on Regeneration through Seeds in Lime (Citrus aurantifolia)
}

\author{
Khalid Akhundzada, P. Venkatesha Murthy, \\ M. Venugopala Reddy* and B. N. Sathyanarayana
}

\begin{abstract}
Department of Horticulture, University of Agricultural Sciences, GKVK, Bengaluru, Karnataka-560065, India
\end{abstract}

*Corresponding author

\section{A B S T R A C T}

\section{Ke ywords \\ BAP, Kinetin, NAA, Lime seed, Germination \\ Article Info \\ Accepted: \\ 15 July 2020 Available Online: 10 August 2020}

In vitro regeneration through seeds were studied in lime (Citrus aurantifolia) by culturing lime seeds on MS media supplemented with BAP (1, 3 and $5 \mathrm{mg} / \mathrm{l})$, Kinetin (1, 3 and $5 \mathrm{mg} / \mathrm{l})$ and their combination with NAA $(0.1,0.5$ and $1 \mathrm{mg} / \mathrm{l})$. In reference to increased germination percentage, mean shoot length, mean number of leaves per explant and mean root length, among BAP and Kinetin concentrations, BAP $3 \mathrm{mg} / \mathrm{l}$ was found to be the best treatment. In terms of cytokinins and NAA combine effect, BAP $5 \mathrm{mg} / \mathrm{l}+$ NAA $0.5 \mathrm{mg} / \mathrm{l}$ and Kinetin 3 $\mathrm{mg} / \mathrm{l}+\mathrm{NAA}$ at $0.1 \mathrm{mg} / \mathrm{l}$ were found to be the best combinations. Survival was 100 per cent when seeds were used as explant material.

\section{Introduction}

Citrus fruits occupy a place of considerable importance in the fruit economy of the country. Citrus fruits are economically important with a large scale production of both the fresh fruit and processed products. They belong to the family Rutaceae of Sapindales order, which consists 140 genera and 1300 species. Citrus fruits are recognized as an important component of the human diet, providing a variety of constituents important to human nutrition, including vitamin $\mathrm{C}$, folic acid, potassium, flavonoids, coumarins, pectin and dietary fibres. Citrus are propagated by both sexual and asexual methods. Most of the commercial varieties are propagated by various asexual methods like Cutting, Grafting and Budding. Usually Seed propagation is practiced in the case of limes and to produce rootstocks for budding purposes, in vitro seedlings are also used for micro grafting. Hence the present study was conducted to investigate into the invitro responses of lime in particular reference to seed as an explant source and could serve as a source of juvenile vegetative explants. 


\section{Materials and Methods}

Lime fruits were procured from local market and brought to Plant Tissue Culture Laboratory, University of Agricultural Sciences, GKVK, Bengaluru.

\section{Explant preparation}

Seeds were extracted from Fruits after treating them in isopropyl alcohol (100\% concentrations) for two hours inside laminar air flow chamber.

\section{Preparation for aseptic culture}

The culture bottles and equipment (forceps, scalpel etc.,) required for initiation were washed thoroughly and rinsed with distilled water, The media bottles were tightened with magenta caps and autoclaved at $121^{\circ} \mathrm{C}$ temperature for 20 minutes. Initially before the use of the LAF (laminar air flow chamber), the working surface was sterilized by wiping the surface of cabinet with 70 per cent alcohol. The UV lamp was switched on for 15 minutes.

The explants transfer was carried out under aseptic conditions inside laminar air flow chamber. The equipment and hands were washed using 70 per cent of alcohol, and the forceps and scalpels were sterilized with glass bead sterilization while transfer of explant onto the culture medium. The culture bottles were swabbed with 70 per cent alcohol before taking into the LAF. The explants were kept on brown paper for cultural operations.

\section{Culture incubation conditions}

All the cultures were incubated in a growth room at a temperature of around $23-27{ }^{\circ} \mathrm{C}$ under a photoperiodic of 12 hours of light provided by white fluorescent tubes.

\section{Experimental details}

Standardization of cytokinin (BAP and Kinetin) concentrations for regeneration through seeds in lime (Citrus aurantifolia).

Standardizing the combined effect of BAP + NAA on regeneration through seeds in lime (Citrus aurantifolia).

Standardizing the combined effect of Kinetin + NAA on regeneration through seeds in lime (Citrus aurantifolia) (Table 1-3).

\section{Results and Discussion}

\section{Germination percentage}

Among all the treatments, Media containing BAP at $3 \mathrm{mg} / \mathrm{l}$ (Table 4), combination of BAP at $5 \mathrm{mg} / \mathrm{l}+\mathrm{NAA}$ at $0.5 \mathrm{mg} / \mathrm{l}$ (Table 5) and Kinetin $3 \mathrm{mg} / \mathrm{l}$ + NAA $0.1 \mathrm{mg} / \mathrm{l}$ (Table 6) were found the best, with a significantly higher per cent (100)of germination obtained, in comparison with media without PGR.

\section{Mean length of shoots}

Among the growth regulators used, media supplemented with BAP at $3 \mathrm{mg} / \mathrm{l}$ (Table 4), BAP $1 \mathrm{mg} / \mathrm{l}+$ NAA $0.1 \mathrm{mg} / \mathrm{l}$ (Table 5) and Kinetin $3 \mathrm{mg} / \mathrm{l}$ + NAA $0.1 \mathrm{mg} / \mathrm{l}$ (Table 6) were found to be the best for increasing the mean shoot length of the explants to an extent of $7.45 \mathrm{~cm}, 4.36 \mathrm{~cm}$ and $3.45 \mathrm{~cm}$ respectively, which were significantly better than control.

\section{Mean length of root}

Media supplemented with Kinetin at $3 \mathrm{mg} / \mathrm{l}$ (Table 4), BAP $1 \mathrm{mg} / \mathrm{l}$ + NAA $0.1 \mathrm{mg} / \mathrm{l}$ (Table 5) and Kinetin $1 \mathrm{mg} / \mathrm{l}+$ NAA $1.0 \mathrm{mg} / \mathrm{l}$ (Table 6) were found to be the best treatments, with increased mean root length of $1.47 \mathrm{~cm}, 2.66 \mathrm{~cm}$ and $2.90 \mathrm{~cm}$ respectively, compared with control which was $0.12 \mathrm{~cm}$. 
Table.1 BAP and Kinetin used for regeneration

\begin{tabular}{|c|c|c|c|}
\hline Treatments & Plant Growth regulators & \multicolumn{2}{|c|}{ Concentration } \\
\cline { 3 - 4 } & $\mathbf{m g} / \mathbf{l}$ & $\boldsymbol{\mu M}$ \\
\hline $\mathbf{T}_{\mathbf{0}}$ & - & - & - \\
\hline $\mathbf{T}_{\mathbf{1}}$ & BAP & 1.0 & 4.44 \\
\hline $\mathbf{T}_{\mathbf{2}}$ & & 3.0 & 13.32 \\
\hline $\mathbf{T}_{\mathbf{3}}$ & & 5.0 & 22.20 \\
\hline $\mathbf{T}_{\mathbf{4}}$ & KINETIN & 1.0 & 4.64 \\
\hline $\mathbf{T}_{\mathbf{5}}$ & & 3.0 & 13.94 \\
\hline $\mathbf{T}_{\mathbf{6}}$ & & 5.0 & 23.23 \\
\hline
\end{tabular}

Table.2 BAP + NAA used for regeneration

\begin{tabular}{|c|c|c|c|}
\hline \multirow{2}{*}{ Treatments } & Plant Growth regulators & \multicolumn{2}{|c|}{ Concentration } \\
\cline { 3 - 4 } & & $\mathbf{m g} / \mathbf{l}$ & $\boldsymbol{\mu M}$ \\
\hline $\mathbf{T}_{\mathbf{0}}$ & - & - & - \\
\hline $\mathbf{T}_{\mathbf{1}}$ & $\mathrm{BAP}(1 \mathrm{mg} / \mathrm{l})+\mathrm{NAA}$ & $1.0+0.1$ & $4.44+0.53$ \\
\hline $\mathbf{T}_{\mathbf{2}}$ & & $1.0+0.5$ & $4.44+2.68$ \\
\hline $\mathbf{T}_{\mathbf{3}}$ & & $1.0+1.0$ & $4.44+5.37$ \\
\hline $\mathbf{T}_{\mathbf{4}}$ & $\mathrm{BAP}(3 \mathrm{mg} / \mathrm{l})+\mathrm{NAA}$ & $3.0+0.1$ & $13.32+0.53$ \\
\hline $\mathbf{T}_{\mathbf{5}}$ & & $3.0+0.5$ & $13.32+2.68$ \\
\hline $\mathbf{T}_{\mathbf{6}}$ & & $3.0+1.0$ & $13.32+5.37$ \\
\hline $\mathbf{T}_{\mathbf{7}}$ & $\mathrm{BAP}(5 \mathrm{mg} / \mathrm{l})+\mathrm{NAA}$ & $5.0+0.1$ & $22.20+0.53$ \\
\hline $\mathbf{T}_{\mathbf{8}}$ & & $5.0+0.5$ & $22.20+2.68$ \\
\hline $\mathbf{T}_{\mathbf{9}}$ & & $5.0+1.0$ & $22.20+5.37$ \\
\hline
\end{tabular}

Table.3 Kinetin + NAA used for regeneration

\begin{tabular}{|c|c|c|c|}
\hline \multirow{2}{*}{ Treatments } & Plant Growth regulators & \multicolumn{2}{|c|}{ Concentration } \\
\cline { 3 - 4 } & - & $\mathrm{mg} / \mathrm{l}$ & $\mu \mathrm{M}$ \\
\hline $\mathbf{T}_{\mathbf{0}}$ & - & - & - \\
\hline $\mathbf{T}_{\mathbf{1}}$ & KINETIN + NAA & $1.0+0.1$ & $4.64+0.53$ \\
\hline $\mathbf{T}_{\mathbf{2}}$ & & $1.0+0.5$ & $13.94+2.68$ \\
\hline $\mathbf{T}_{\mathbf{3}}$ & & $1.0+1.0$ & $23.23+5.37$ \\
\hline $\mathbf{T}_{\mathbf{4}}$ & KINETIN + NAA & $3.0+0.1$ & $4.64+0.53$ \\
\hline $\mathbf{T}_{\mathbf{5}}$ & & $3.0+0.5$ & $13.94+2.68$ \\
\hline $\mathbf{T}_{\mathbf{6}}$ & & $3.0+1.0$ & $23.23+5.37$ \\
\hline $\mathbf{T}_{\mathbf{7}}$ & KINETIN + NAA & $5.0+0.1$ & $4.64+0.53$ \\
\hline $\mathbf{T}_{\mathbf{8}}$ & & $5.0+0.5$ & $13.94+2.68$ \\
\hline $\mathbf{T}_{\mathbf{9}}$ & & $5.0+1.0$ & $23.23+5.37$ \\
\hline
\end{tabular}


Table.4 BAP and Kinetin used

\begin{tabular}{|c|c|c|c|c|c|}
\hline Treatments & $\begin{array}{l}\text { Germination } \\
\text { percentage }\end{array}$ & $\begin{array}{l}\text { shoot } \\
\text { length }\end{array}$ & $\begin{array}{l}\text { length of } \\
\text { root }\end{array}$ & $\begin{array}{l}\text { Number of } \\
\text { leaves }\end{array}$ & $\begin{array}{c}\text { Survival } \\
\text { percentage }\end{array}$ \\
\hline $\mathbf{T}_{\mathbf{0}}$ & 20 & 0.8 & 0.12 & 1.30 & 100.00 \\
\hline$T_{1}$ & 50 & 0.73 & 0.20 & 1.30 & 100.00 \\
\hline $\mathbf{T}_{2}$ & 100 & 7.45 & 1.20 & 7.90 & 100.00 \\
\hline $\mathbf{T}_{3}$ & 80 & 1.8 & 0.33 & 2.50 & 100.00 \\
\hline$T_{4}$ & 60 & 0.45 & 0.70 & 1.30 & 100.00 \\
\hline$T_{5}$ & 30 & 2.5 & 1.47 & 1.90 & 100.00 \\
\hline$T_{6}$ & 40 & 0.8 & 0.90 & 2.00 & 100.00 \\
\hline F@ @ & $*$ & $*$ & $*$ & $*$ & NS \\
\hline CD & 47.7 & 2.2 & 0.40 & 2.60 & - \\
\hline SEm \pm & 16.4 & 0.8 & 0.12 & 0.94 & - \\
\hline \multicolumn{6}{|c|}{$\begin{array}{lr}\mathbf{T}_{1}: \text { BAP } 1 \mathrm{mg} / \mathrm{l} & \mathrm{T}_{4}: \text { Kinetin } 1 \mathrm{mg} / \mathrm{l} \\
\mathbf{T}_{2}: \text { BAP } 3 \mathrm{mg} / \mathrm{l} & \mathrm{T}_{5}: \text { Kinetin } 3 \mathrm{mg} / \\
\mathrm{T}_{3}: \text { BAP } 5 \mathrm{mg} / \mathrm{l} & \mathrm{T}_{6}: \text { Kinetin } 5 \mathrm{mg} / \mathrm{l} \\
\text { NS : Non significant } & * \text { : Significant }\end{array}$} \\
\hline
\end{tabular}

Table.5 BAP combined with NAA used

\begin{tabular}{|c|c|c|c|c|c|}
\hline Treatments & $\begin{array}{l}\text { Germination } \\
\text { percentage }\end{array}$ & $\begin{array}{l}\text { shoot length } \\
(\mathrm{cm})\end{array}$ & root length & $\begin{array}{c}\text { Number of } \\
\text { leaves }\end{array}$ & $\begin{array}{c}\text { Survival } \\
\text { percentage }\end{array}$ \\
\hline $\mathbf{T}_{\mathbf{0}}$ & 20.00 & 0.8 & 0.12 & 1.3 & 100.00 \\
\hline$T_{1}$ & 70.00 & 4.36 & 2.50 & 4.2 & 100.00 \\
\hline $\mathbf{T}_{2}$ & 70.00 & 3.32 & 0.83 & 4.1 & 100.00 \\
\hline$T_{3}$ & 60.00 & 2.31 & 0.32 & 3.5 & 100.00 \\
\hline $\mathbf{T}_{4}$ & 30.00 & 0.2 & 0.15 & 0.3 & 100.00 \\
\hline$T_{5}$ & 40.00 & 0.8 & 0.26 & 2.6 & 100.00 \\
\hline $\mathbf{T}_{6}$ & 40.00 & 0.55 & 0.00 & 1.5 & 100.00 \\
\hline $\mathbf{T}_{7}$ & 30.00 & 0.85 & 0.55 & 0.65 & 100.00 \\
\hline $\mathbf{T}_{8}$ & 100.00 & 3.66 & 0.90 & 6.8 & 100.00 \\
\hline $\mathbf{T}_{9}$ & 40.00 & 0.55 & 0.12 & 1.4 & 100.00 \\
\hline F@ $9 \%$ & $*$ & $*$ & $*$ & $*$ & NS \\
\hline CD & 50.72 & 2.26 & 0.93 & 3.07 & - \\
\hline SEm \pm & 17.74 & 0.80 & 0.33 & 1.09 & - \\
\hline 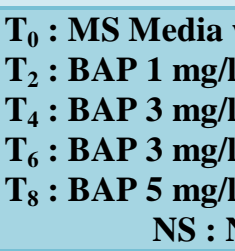 & $\begin{array}{l}\text { ithout plant gros } \\
\text { + NAA } 0.5 \mathrm{mg} / \mathrm{l} \\
\text { + NAA } 0.1 \mathrm{mg} / \mathrm{l} \\
\text { + NAA } 1.0 \mathrm{mg} / \mathrm{l} \\
\text { + NAA } 0.5 \mathrm{mg} / \mathrm{l} \\
\text { on significant }\end{array}$ & $\begin{array}{r}\text { vth regulators } \\
\mathbf{T}_{3} \\
\mathbf{T}_{5} \\
\mathbf{T}_{7} \\
\mathbf{T}_{9}\end{array}$ & $\begin{array}{l}\mathrm{T}_{1}: \text { BAP } 1 \mathrm{mg} / \mathrm{l} \\
: \text { BAP } 1 \mathrm{mg} / \mathrm{l}+ \\
: \text { BAP } 3 \mathrm{mg} / \mathrm{l}+ \\
: \text { BAP } 5 \mathrm{mg} / \mathrm{l}+ \\
\text { : BAP } 5 \mathrm{mg} / \mathrm{l}+ \\
\text { * : Significa }\end{array}$ & $\begin{array}{l}\text { + NAA } 0.1 \mathrm{mg} \text { / } \\
\text { NAA } 1.0 \mathrm{mg} / \mathrm{l} \\
\text { NAA } 0.5 \mathrm{mg} / \mathrm{l} \\
\text { NAA } 0.1 \mathrm{mg} / \mathrm{l} \\
\text { vAA } 1.0 \mathrm{mg} / \mathrm{l} \\
\text { it }\end{array}$ & \\
\hline
\end{tabular}


Table.6 BAP combined with NAA used

\begin{tabular}{|c|c|c|c|c|c|}
\hline Treatments & $\begin{array}{l}\text { Germination } \\
\text { percentage }\end{array}$ & $\begin{array}{l}\text { shoot length } \\
(\mathrm{cm})\end{array}$ & $\begin{array}{l}\text { root length } \\
\qquad(\mathrm{cm})\end{array}$ & $\begin{array}{l}\text { Number } \\
\text { of leaves }\end{array}$ & $\begin{array}{c}\text { Survival } \\
\text { percentage }\end{array}$ \\
\hline $\mathbf{T}_{\mathbf{0}}$ & 20.00 & 0.80 & 0.12 & 1.33 & 100.00 \\
\hline $\mathbf{T}_{1}$ & 60.00 & 3.00 & 1.65 & 3.10 & 100.00 \\
\hline $\mathbf{T}_{2}$ & 50.00 & 0.90 & 1.05 & 1.00 & 100.00 \\
\hline $\mathbf{T}_{\mathbf{3}}$ & 60.00 & 1.20 & 2.90 & 2.10 & 100.00 \\
\hline $\mathbf{T}_{4}$ & 90.00 & 3.45 & 2.40 & 9.60 & 100.00 \\
\hline $\mathbf{T}_{5}$ & 20.00 & 0.90 & 1.10 & 1.50 & 100.00 \\
\hline $\mathbf{T}_{6}$ & 10.00 & 1.20 & 0.00 & 0.40 & 100.00 \\
\hline $\mathbf{T}_{7}$ & 70.00 & 1.70 & 1.25 & 2.90 & 100.00 \\
\hline $\mathbf{T}_{8}$ & 90.00 & 2.73 & 2.50 & 5.50 & 100.00 \\
\hline $\mathbf{T}_{9}$ & 40.00 & 0.55 & 0.55 & 0.60 & 100.00 \\
\hline F@ $9 \%$ & $*$ & $*$ & $*$ & $*$ & NS \\
\hline CD & 47.20 & 2.01 & 1.80 & 5.50 & - \\
\hline SEm \pm & 16.73 & 0.71 & 0.64 & 1.95 & - \\
\hline \multicolumn{6}{|c|}{$\begin{array}{cc}T_{0}: \text { MS Media without PGR } & T_{1}: \text { Kinetin } 1 \mathrm{mg} / \mathrm{l}+\text { NAA } 0.1 \mathrm{mg} / \mathrm{l} \\
\mathrm{T}_{2}: \text { Kinetin } 1 \mathrm{mg} / \mathrm{l}+\text { NAA } 0.5 \mathrm{mg} / \mathrm{l} & \mathrm{T}_{3}: \text { Kinetin } 1 \mathrm{mg} / \mathrm{l}+\mathrm{NAA} 1.0 \mathrm{mg} / \mathrm{l} \\
\mathrm{T}_{4}: \text { Kinetin } 3 \mathrm{mg} / \mathrm{l}+\text { NAA } 0.1 \mathrm{mg} / \mathrm{l} & \mathrm{T}_{5}: \text { Kinetin } 3 \mathrm{mg} / \mathrm{l}+\text { NAA } 0.5 \mathrm{mg} / \mathrm{l} \\
\mathrm{T}_{6}: \text { Kinetin } 3 \mathrm{mg} / \mathrm{l}+\text { NAA } 1.0 \mathrm{mg} / \mathrm{l} & \mathrm{T}_{7}: \text { Kinetin } 5 \mathrm{mg} / \mathrm{l}+\text { NAA } 0.1 \mathrm{mg} / \mathrm{l} \\
\mathrm{T}_{8}: \text { Kinetin } 5 \mathrm{mg} / \mathrm{l}+\text { NAA } 0.5 \mathrm{mg} / \mathrm{l} & \mathrm{T}_{9}: \text { Kinetin } 5 \mathrm{mg} / \mathrm{l}+\text { NAA } 1.0 \mathrm{mg} / \mathrm{l} \\
\quad \text { NS }: \text { Non significant } & \text { * : Significant }\end{array}$} \\
\hline
\end{tabular}

Plate.1 Callus obtained from lime seed explants with BA 5 mg/L + NAA 0.5 mg/L

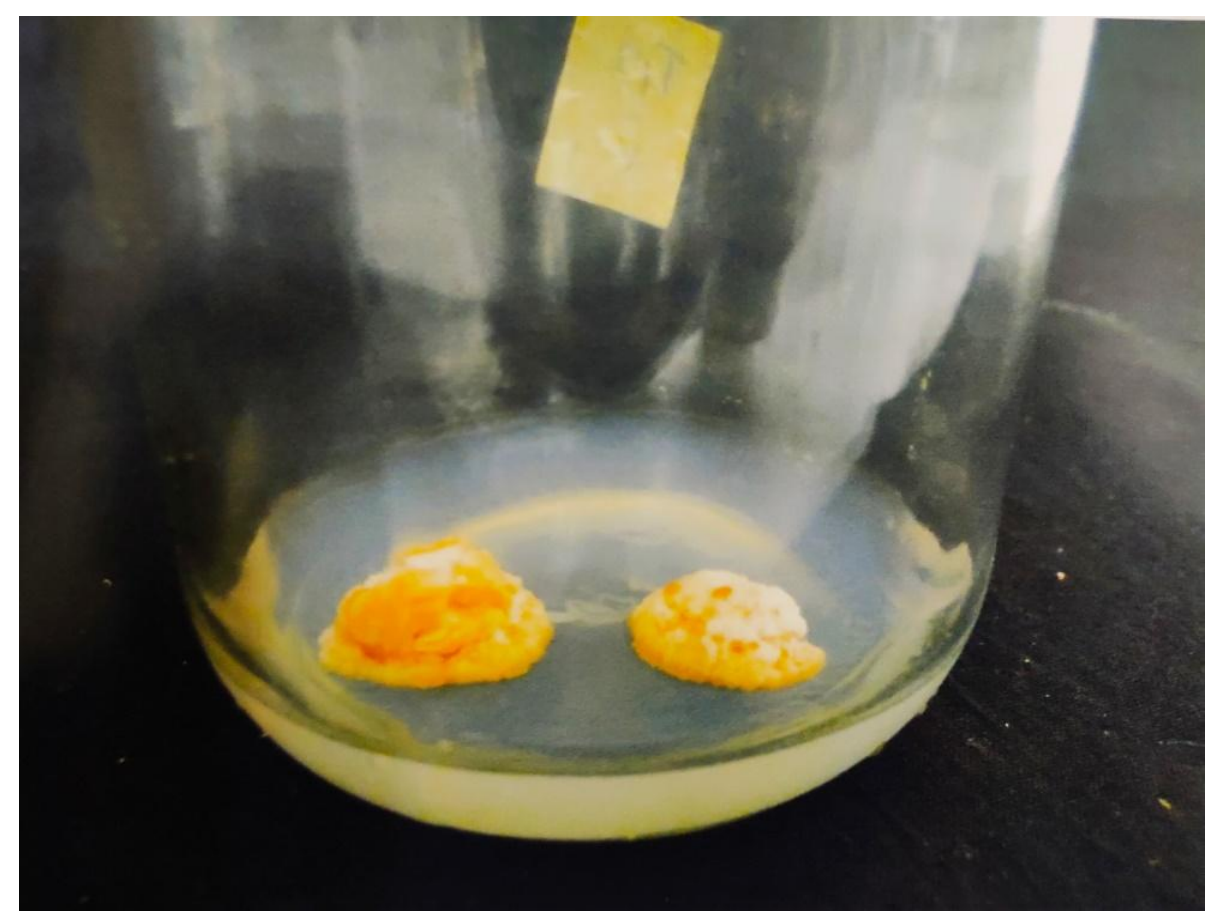


Plate.2 Lime seedlings obtained with BA $3 \mathrm{mg} / \mathrm{L}$

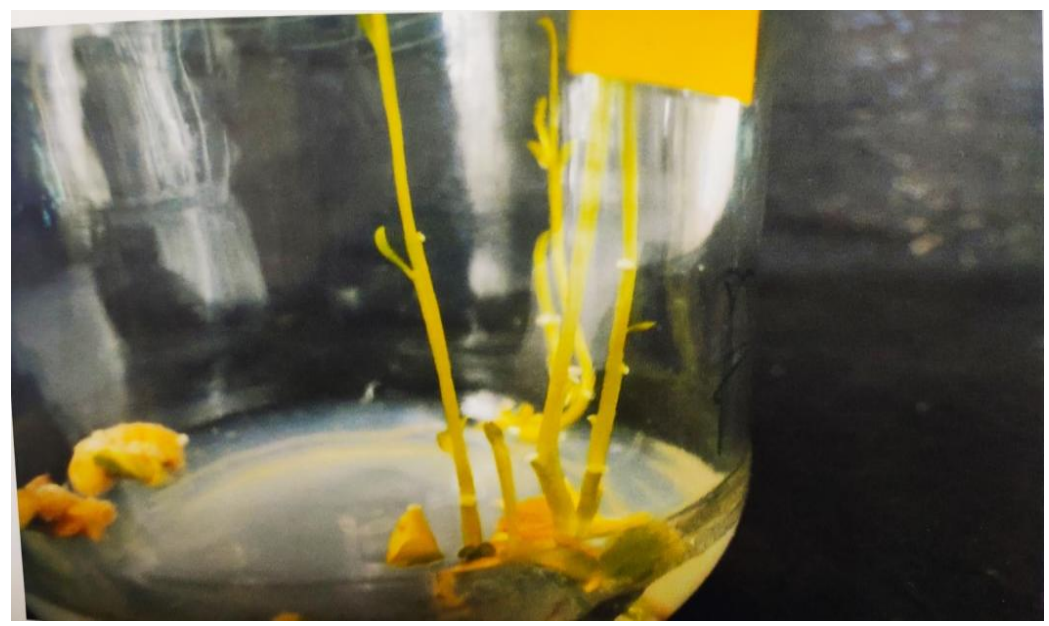

Plate.3 Seedling roots observed with Kinetin 3mg/L + NAA 0.1 mg/L

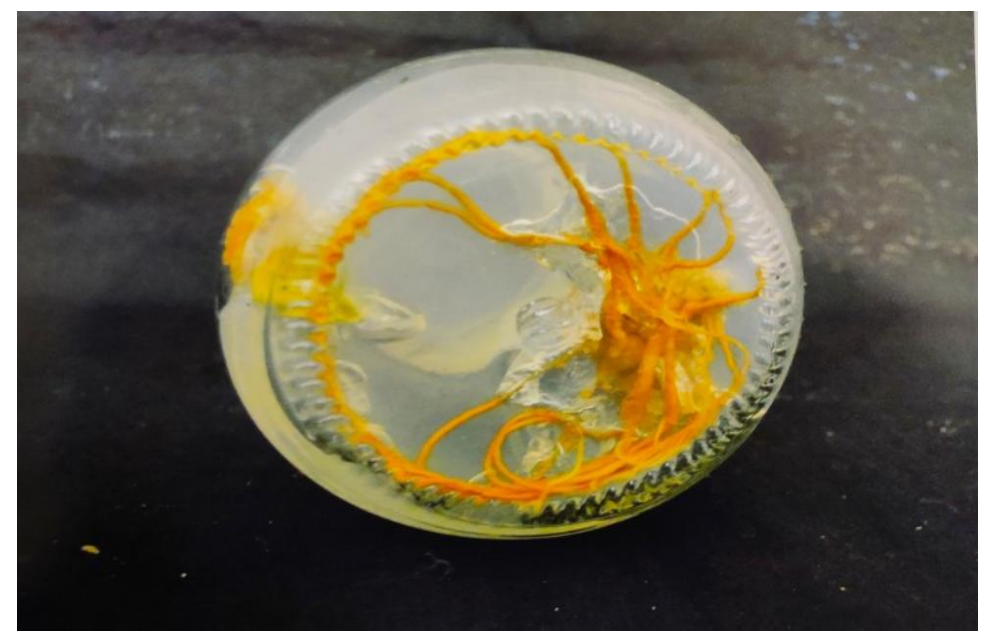

Plate.4 Mandarin seedlings obtained with Kinetin 5mg/L + NAA 0.5mg/L

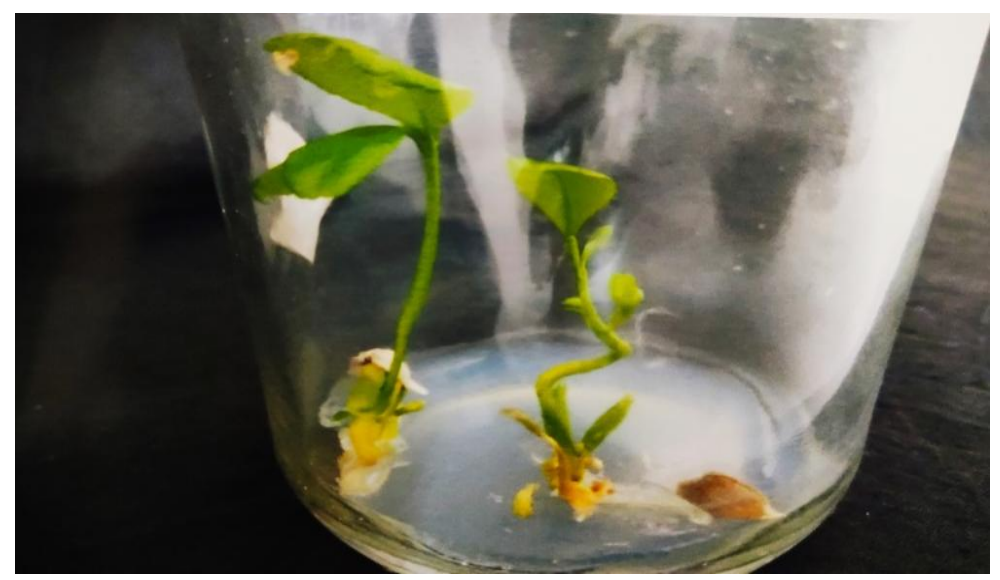




\section{Mean number of leaves/explant}

As it has been observed with other parameters in this experiment, media with BAP at $3 \mathrm{mg} / \mathrm{l}$ (Table 4), BAP at $5 \mathrm{mg} / \mathrm{l}+\mathrm{NAA}$ at $0.5 \mathrm{mg} / \mathrm{l}$ (Table 5) and Kinetin $3 \mathrm{mg} / \mathrm{l}+$ NAA $0.1 \mathrm{mg} / \mathrm{l}$ (Table 6) were again the best treatments in increasing the mean number of leaves obtained per explant to an extent of 7.9, 6.8 and 9.6 respectively, which were significantly better among all the PGR concentrations.

\section{Survival percentage}

Survival was 100 per cent when seeds were used as explant material (Table 4-6).

Standardization of cytokinin (BAP and Kinetin) and their concentration for regeneration through seeds in lime (Citrus aurantifolia)

In the present investigation, BAP at $3 \mathrm{mg} / \mathrm{l}$ were found to be the best treatments in Lime (Citrus aurantifolia), in this growth aspects such as germination percentage, mean number of leaves/explant and mean length of shoots. This kind of response is quite a common phenomenon with growth regulators such as cytokinins and in particular reference BAP and Kinetin. Similarly, in Citrus limon, the highest regeneration was reported on Murashige and Tucker (M.T.) medium supplemented with BAP at $3.5 \mathrm{mg} / \mathrm{l}$ (Anna et al., 2015), BAP at $2.5 \mathrm{mg} / \mathrm{l}$ (Mekdes et al., 2016) and BAP at 3 mg/l (Rathore et al., 2007).

In the previous experiment (Table 4) BAP had been included as a growth regulator. The presence of Cytokinins (BAP and Kinetin) had significantly increased germination percentage, length of seedling and others. Here in the present experiment when NAA was included along with BAP (BAP $5 \mathrm{mg} / \mathrm{l}+$ NAA at $0.5 \mathrm{mg} / \mathrm{l}$ ) and Kinetin (Kinetin $3 \mathrm{mg} / \mathrm{l}$ + NAA $0.1 \mathrm{mg} / \mathrm{l})$,all the parameters such germination percentage, number of leaves per explant, mean length of shoots has been much enhanced, this is probably because the role of NAA in influencing the production of GA in the plant system (Cleon and Frank, 2006).Similarly Singh et al., (1994) observed higher shoot induction in Citrus limon and Citrus reticulata, when they were cultured on MS media supplemented with Kinetin at 1 $\mathrm{mg} / \mathrm{l}+\mathrm{NAA}$ at $0.1 \mathrm{mg} / \mathrm{l}$. In respect of grapefruit (Citrus paradasi), a combination of BAP and NAA ( $3 \mathrm{mg} / \mathrm{l}+0.5 \mathrm{mg} / \mathrm{l})$ has been reported to be the best combination in enhancing bud proliferation (Babita and Harshad, 2015).

In conclusion the seeds can be used as planting material for in-vitro regeneration of lime. Different combinations of plant growth regulators have varied effects on the plant tissue. In this investigation the best results were obtained when seeds were cultured on MS media supplemented with BAP at $3 \mathrm{mg} / \mathrm{l}$ followed by Kinetin at $3 \mathrm{mg} / \mathrm{l}+\mathrm{NAA}$ at 0.1 $\mathrm{mg} / \mathrm{l}$.

\section{References}

Anna, K. P., Jacek P. and Ewa S., 2015, In vitro regeneration induced in leaf explants of Citrus limon L. burm cv. 'Primofiore'. Acta Sci. Pol., 14(4): 143-153.

Babita, R. and Harshad, M. P., 2015, Effect of explant type and different plant growth regulators on callus induction and plantlet regeneration in grapefruit, Citrus decumana var. paradise (Macfad.).Ind. J. Appl. Res., 5(7): 445448.

Cleon, W. R. and Frank, B. S., 2006, Plantphysiology, Edn 4, CBS Publishers and Distributers, 204-206.

Mekdes, F., Temesgen, M. and Kassahun, B., Optimization of sucrose, plant hormones and photoperiod for in vitro 
propagation of lemon ( $C$. limon) and macrophylla ( $C$. macrophylla) using shoot tip.Adv. L. Sci. Tech., 47: 31-39.

Rathore, J. S., Rathore, M. S., Singh, R. P., singh, M. and Shekhawat, M. S., 2007, Micropropagation of mature tree of Citrus limon. Ind. J. Biotech., 6: 239-
244.

Singh, S., Ray, B. K., Bhattacharyya, S. and Deka, P. C., 1994, In-vitro propagation of Citrus reticulate Blanco and Citrus limon Burm. Hort. Sci., 29(3): 214216.

\section{How to cite this article:}

Khalid Akhundzada, P. Venkatesha Murthy, M. Venugopala Reddy and Sathyanarayana, B. N. 2020. Standardization of Cytokinins (BAP and Kinetin) Concentrations and their Combination with NAA on Regeneration through Seeds in Lime (Citrus aurantifolia). Int.J.Curr.Microbiol.App.Sci. 9(08): 1245-1252. doi: https://doi.org/10.20546/ijcmas.2020.908.140 